\title{
Association between intake of non-sugar sweeteners and health outcomes: systematic review and meta-analyses of randomised and non-randomised controlled trials and observational studies
}

\author{
Ingrid Toews, ${ }^{1}$ Szimonetta Lohner, ${ }^{2}$ Daniela Küllenberg de Gaudry, ${ }^{1}$ Harriet Sommer, ${ }^{1,3}$ \\ Joerg J Meerpohl ${ }^{1,4}$
}

${ }^{1}$ Institute for Evidence in

Medicine (for Cochrane

Germany Foundation), Medical

Centre of the University of

Freiburg, Faculty of Medicine,

University of Freiburg,

Breisacher Straße 153, 79110

Freiburg, Germany

${ }^{2}$ Cochrane Hungary, Clinical

Centre of the University of Pécs,

Medical School, University of

Pécs, Pécs, Hungary

${ }^{3}$ Institute for Medical Biometry

and Statistics, Medical Centre

of the University of Freiburg,

Faculty of Medicine, University

of Freiburg, Freiburg, Germany

${ }^{4}$ Centre of Epidemiological and

Statistical Research, Sorbonne

Paris Cité, Inserm/Université

Paris Descartes, Cochrane

France, Paris, France

Correspondence to:

J) Meerpohl

Meerpohl@cochrane.de

(ORCID 0000-0002-1333-5403)

Additional material is published

online only. To view please visit

the journal online.

Cite this as: BMJ 2019;364:k4718 http://dx.doi.org/10.1136/bmj.k4718

Accepted: 29 October 2018

\section{ABSTRACT \\ OBJECTIVE}

To assess the association between intake of non-sugar sweeteners (NSS) and important health outcomes in generally healthy or overweight/obese adults and children.

\section{DESIGN}

Systematic review following standard Cochrane review methodology.

\section{DATA SOURCES}

Medline (Ovid), Embase, Cochrane CENTRAL, WHO

International Clinical Trials Registry Platform,

Clinicaltrials.gov, and reference lists of relevant publications.

\section{ELIGIBILITY CRITERIA FOR SELECTING STUDIES}

Studies including generally healthy adults or children with or without overweight or obesity were eligible. Included study designs allowed for a direct comparison of no intake or lower intake of NSS with higher NSS intake. NSSs had to be clearly named, the dose had to be within the acceptable daily intake, and the intervention duration had to be at least seven days.

\section{MAIN OUTCOME MEASURES}

Body weight or body mass index, glycaemic control, oral health, eating behaviour, preference for sweet taste, cancer, cardiovascular disease, kidney disease, mood, behaviour, neurocognition, and adverse effects.

RESULTS

The search resulted in 13941 unique records. Of 56 individual studies that provided data for this review,

\section{WHAT IS ALREADY KNOWN ON THIS TOPIC}

Studies have suggested an association between the use of non-sugar sweeteners and health outcomes (such as body weight, diabetes, cancer, and oral health)

However, evidence for health effects due to the use of non-sugar sweeteners is conflicting

Existing reviews on non-sugar sweeteners and health outcomes have limitations in scope and currency

\section{WHAT THIS STUDY ADDS}

In this comprehensive systematic review, a broad range of health outcomes were investigated to determine a possible association with non-sugar sweetener use in a generally healthy population

There was no compelling evidence to indicate important health benefits of nonsugar sweetener use on a range of health outcomes

Potential harms from the consumption of non-sugar sweeteners could not be excluded

35 were observational studies. In adults, evidence of very low and low certainty from a limited number of small studies indicated a small beneficial effect of NSSs on body mass index (mean difference -0.6 , $95 \%$ confidence interval -1.19 to -0.01 ; two studies, $\mathrm{n}=174)$ and fasting blood glucose $(-0.16 \mathrm{mmol} / \mathrm{L}$, -0.26 to -0.06 ; two, $n=52$ ). Lower doses of NSSs were associated with lower weight gain $(-0.09 \mathrm{~kg}$, -0.13 to -0.05 ; one, $n=17$ 934) compared with higher doses of NSSs (very low certainty of evidence). For all other outcomes, no differences were detected between the use and non-use of NSSs, or between different doses of NSSs. No evidence of any effect of NSSs was seen on overweight or obese adults or children actively trying to lose weight (very low to moderate certainty). In children, a smaller increase in body mass index z score was observed with NSS intake compared with sugar intake $(-0.15,-0.17$ to -0.12 ; two, $n=528$, moderate certainty of evidence), but no significant differences were observed in body weight $(-0.60 \mathrm{~kg},-1.33$ to 0.14 ; two, $n=467$, low certainty of evidence), or between different doses of NSSs (very low to moderate certainty).

\section{CONCLUSIONS}

Most health outcomes did not seem to have differences between the NSS exposed and unexposed groups. Of the few studies identified for each outcome, most had few participants, were of short duration, and their methodological and reporting quality was limited; therefore, confidence in the reported results is limited. Future studies should assess the effects of NSSs with an appropriate intervention duration. Detailed descriptions of interventions, comparators, and outcomes should be included in all reports.

\section{SYSTEMATIC REVIEW REGISTRATION}

Prospero CRD42017047668.

\section{Introduction}

Growing concerns about health and quality of life have encouraged people to adapt healthy lifestyles and avoid the consumption of food rich in sugars, salt, or fat to prevent obesity and other non-communicable diseases. With increased consumer interest in reducing energy intake, food products containing nonsugar sweeteners (NSSs) rather than simple sugars (monosaccharides and disaccharides) have become increasingly popular. ${ }^{1}$ Replacement of sugars with NSSs bears promise of health benefits primarily by reducing the contribution of sugars to daily calorie intake and thus reducing the risk of unhealthy weight 
gain. ${ }^{2-4}$ However, evidence for health effects due to use of NSSs is conflicting. While some studies report an association between NSS use and reduced risk of type 2 diabetes, overweight, and obesity (thus suggesting a benefit for general health and the management of diabetes), ${ }^{56}$ other studies suggest that NSS use could increase the risk of overweight, diabetes, and cancer. ${ }^{7}$ Further investigations are needed to clarify the benefits and harms of NSS consumption. Therefore, the objective of our review was to investigate the health effects of NSSs in adults and children.

\section{Description of the exposure or intervention of interest}

Most NSSs so far have been synthesised, but through research and development in food chemistry and processing, the number of natural NSS compounds is increasing. ${ }^{8}$ NSSs differ from sugars not only in their taste properties, but also in how the body metabolises them ${ }^{9}$ and how they in turn affect physiological processes. ${ }^{10}$ NSSs are generally sweeter than sucrose, but contain far fewer or no calories. Each sweetener is unique in its sweetness intensity, persistence of the sweet taste, coating of the teeth, and aftertaste effect. $^{11}$

The definitions and terminology for NSSs vary. In some cases, the term "artificial sweeteners" is used as a synonym for NSSs, in other cases as a subcategory. In this systematic review, we use the term "NSSs" as a category including both artificial sweeteners and naturally occurring non-caloric sweeteners (fig 1). The term "NSSs" is also used by the CODEX Alimentarius (part of the Joint Food and Agriculture Organisation of the United Nations/World Health Organization Food Standards Programme), and this review was conducted in support of guidelines being developed by WHO.

The range of NSSs approved in different countries varies. In the United States, for example, the Food and Drug Administration has approved six NSSs for consumption, ${ }^{12}$ whereas the range of currently approved NSSs in the European Union is wider (eg, including cyclamate). ${ }^{13}$ In general, current evidence supports the safety of several NSSs to be used in foods. ${ }^{14}$ Recognised regulatory bodies have established acceptable daily intakes based on various safety studies. Other NSSs are currently declared as unsafe or have not yet been assessed.

Although many of the NSSs currently being used in foods have been declared safe for consumption at levels below the respective acceptable daily intakes, less is known regarding potential benefits and harms of NSSs

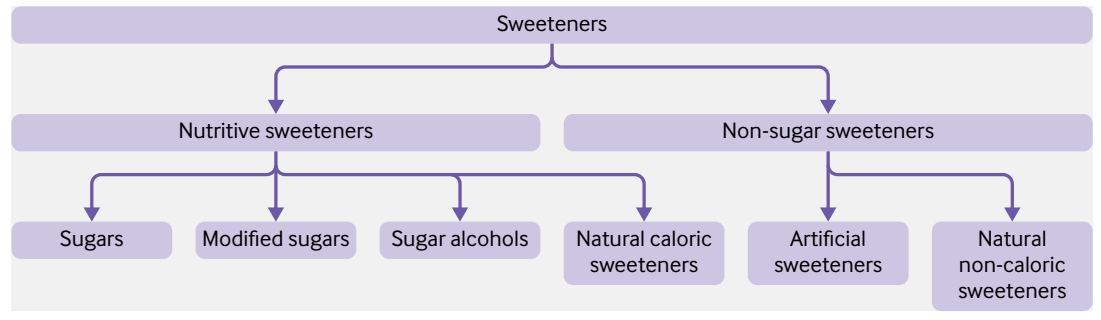

Fig 1 | Types of sweeteners of interest in context within this range of intake, beacuse evidence from studies and reviews is often limited and conflicting. WHO is developing guidance on the use of NSSs by adults and children based on the evidence generated by this systematic review. Following the guidance of the WHO Nutrition Guidance Expert Advisory Group Subgroup on Diet and Health, this review seeks to comprehensively assess the association between commonly consumed NSSs and health by looking at the following research questions:

- In a general adult population, what are the effects of NSS consumption versus no consumption on relevant health outcomes?

- In a general adult population, what are the effects of higher versus lower NSS doses and more frequent versus less frequent NSS consumption on relevant health outcomes?

- In an overweight or obese adult population with explicit intentional weight loss, what are the effects of NSS consumption versus no consumption on relevant health outcomes?

- In a general child population, what are the effects of NSS consumption versus no consumption on relevant outcomes?

- In a general child population, what are the effects of higher versus lower NSS doses and more frequent versus less frequent NSSs consumption on relevant here outcomes?

- In a population of overweight and obese children with explicit intentional weight loss, what are the effects of NSS consumption versus no consumption on relevant outcomes?

\section{Methods}

In accordance with the WHO guideline development process, ${ }^{15}$ we conducted a systematic review and meta-analyses according to the methodological recommendations of the Cochrane Collaboration. ${ }^{16}$ Ethical approval was not required for this research.

\section{Inclusion criteria}

The inclusion and exclusion criteria for this review were established prospectively and were based on their relevance for a WHO global guideline for NSS use by a generally healthy population. We included studies with a general, healthy population of adults ( $\geq 18$ years) or children ( $<18$ years), including those with overweight or obesity. Studies that exclusively included overweight or obese adults or children who were specifically trying to lose weight (that is, weight loss studies) were also included and analysed separately. We excluded studies including diseased populations, in vitro and animal studies. Studies with pregnant women were also excluded.

The interventions and exposures of interest included any type of NSSs, either as an individual intervention or in combination with other NSSs. Interventions or exposures described as "diet sodas," "diet beverages," or "diet soft drinks" were included when the sweeteners used in the products were NSSs and their 
type was sufficiently specified. We excluded studies that did not specify the type of sweetener. Studies that applied concomitant interventions were included as long as the interventions were similar and equally balanced between the intervention and comparator groups to establish fair comparisons. We included studies that reported to use NSSs within the acceptable daily intake as established by the Joint FAO (Food and Agriculture Organization of the United Nations)/ WHO Expert Committee on Food Additives, European Food Safety Authority, or the United States Food and Drug Administration (table 1), or did not report any information on dose. If the acceptable daily intake values differed between the regulatory bodies, we used the higher value as the threshold for inclusion in our review. Studies in which sweetener intake explicitly exceeded the acceptable daily intake were excluded. All studies had to have a minimum intervention duration of seven days.

We included studies that compared the intervention against the intake of any alternative intervention, for example, any other type of caloric or non-caloric sweetener, any type of sugar, no intervention, placebo, or plain water. The outcomes of interest included body weight, oral health, incidence of diabetes, eating behaviour. Secondary outcomes were preference for sweet taste, incidence of any type of cancer, incidence of cardiovascular disease, incidence of chronic kidney disease, incidence of asthma, incidence of allergies, mood, behaviour, and neurocognition.

We included all parallel grouped or crossover (quasi-)randomised controlled trials, and cluster randomised trials. In crossover randomised controlled trials, we considered both phases of the study because the effect of NSS intake is not expected to last long enough to bias the results from the second phase of crossover trials for the outcomes evaluated in this review. Furthermore, we included non-randomised controlled trials ${ }^{21}$ as well as prospective and retrospective cohort studies, case-control studies, and cross sectional studies but analysed them separately. Studies with observational design were included because the possible long term effects of NSSs-for example, on the incidence of non-communicable diseases such as cancer-are generally difficult to assess in randomised controlled trials. We included unpublished and ongoing studies.

\section{Search methods for identification of studies}

The search strategy for this review combined electronic searches and hand searching. For the electronic searches, no date or language restrictions were applied. A systematic literature search in the following databases was conducted last on 25 May 2017 (by SL): Medline, Medline in Process and Medline Daily Update, Embase, and the Cochrane Central Register of Controlled Trials (CENTRAL). To identify ongoing or completed, but unpublished trials, the WHO International Clinical Trials Registry Platform (ICTRP) search portal) as well as ClinicalTrials.gov were searched on 23 November 2017 (by IT). Search strategies are listed in the supplementary file 1 . The reference lists of relevant systematic reviews were screened manually to identify further potentially relevant citations.

\section{Selection of studies}

All titles and abstracts of records identified in the databases and other sources above were screened for eligibility by one researcher (DKdG, SL, or IT). Two review authors independently evaluated full texts of all potentially eligible studies for appropriateness for inclusion without prior consideration of the results (DKdG, SL, IT). Any disagreements were resolved by discussion or feedback from a third author (JJM).

\section{Data extraction and management}

Two review authors independently extracted data and cross checked the extracted information on study characteristics, and included participants, interventions, and reported outcomes using a piloted, standardised data extraction form in the online software Covidence (DKdG, SL, IT). Any differences related to the data extraction were resolved by rechecking the full text of the study or by discussion. If study data were only available from figures, data were extracted by use of the validated software Plot Digitizer (plotdigitizer. sourceforge.net). ${ }^{22}$ When study data were ambiguous or data were not reported in a form that could be used for formal comparison, we contacted the corresponding and first author of the original publication via email.

\begin{tabular}{|c|c|c|c|}
\hline \multirow[b]{2}{*}{ Non-sugar sweetener } & \multicolumn{3}{|c|}{ Acceptable daily intake (mg intake per $\mathrm{kg}$ of body weight) } \\
\hline & $\mathrm{JECFAs}^{17}$ & European Food Safety Authority & US Food and Drug Administration \\
\hline Acesulfame K & 15 & $9^{18}$ & 15 \\
\hline Advantame & 5 & 5 & 32.8 \\
\hline Aspartame & 40 & 40 & 50 \\
\hline Brazzein & - & Not approved & Not approved \\
\hline Cyclamate & 11 & 7 & Not approvedNot approved \\
\hline Neotame & 0.3 & $0-2^{19}$ & 0.30 \\
\hline Saccharin & 15 & 5 & 15 \\
\hline Sucralose & 5 & 15 & 5 \\
\hline Steviol glycosides & 4 & $4^{20}$ & 4 \\
\hline Thaumatin & Not approved & Not specified & Not approved \\
\hline
\end{tabular}




\section{Assessment of risk of bias}

Two review authors independently assessed the risk of bias for each study. Any disagreements were resolved by discussion or a third author (JJM). For the risk of bias assessment of randomised controlled trials, we used the Cochrane risk of bias tool. ${ }^{23}$ For non-randomised controlled trials, we used the ROBINS-I tool (risk of bias in non-randomised studies of interventions). ${ }^{24}$ We planned to create funnel plots when data of 10 or more studies were available to assess the likelihood of dissemination bias. Since none of the meta-analyses included 10 studies or more, a thorough assessment of dissemination bias was not feasible.

\section{Data synthesis}

If not reported, we calculated the risk ratios and their respective 95\% confidence intervals for randomised controlled trials, controlled clinical trials, and cohort studies, as well as odds ratios and their respective 95\% confidence intervals for case-control studies. Mean differences or standardised mean differences with $95 \%$ confidence intervals were calculated for continuous outcomes. We conducted meta-analyses if comparable outcome data from two or more studies were available. In these meta-analyses, we used the random effects model. When baseline and final values were given, we computed changes from baseline. We imputed any missing standard deviation values using an imputed correlation coefficient. ${ }^{25}$ In this review, we used a correlation coefficient of zero. Statistical analyses were conducted by the statistical software $\mathrm{R}$ with the $\mathrm{R}$ package meta and metasens. ${ }^{26}$

\section{Sensitivity analyses}

We tested the robustness of our results using sensitivity analyses. In forest plots, we reported results of analyses with the random effects model as our primary effect estimate. For all meta-analyses, we conducted sensitivity analyses using the fixed effect model. In most sensitivity analyses with the fixed effect model, the effects were more precise (narrower 95\% confidence intervals) and consequently statistically significant at times, compared with analyses using the random effects model. However, given the clinical heterogeneity of the included studies, these were judged to not be appropriate, and therefore the results are not reported in detail. We found only one study with low risk of bias; thus, an analysis of studies with a low risk of bias only was not feasible. Study populations were divided into participants aged 18 years and older and those aged younger than 18 years in sensitivity analyses so that the effect of NSSs on children only and adults only could be analysed.

\section{Assessment of the certainty of the evidence}

We used the GRADE approach (grading of recommendations assessment, development, and evaluation) to assess the certainty of the evidence for the most relevant, available measures of all critical and important outcomes. ${ }^{27}$ According to the GRADE approach, we classified the certainty of evidence in four categories: high, moderate, low, and very low certainty of evidence. The GRADE certainty assessment per outcome was documented in GRADE evidence profiles, together with the pooled effects for the interventions. We used GRADEpro GDT online software ${ }^{28}$ to compile the evidence profiles. Assessments of the certainty of evidence for all outcomes were reviewed with the WHO Nutrition Guidance Expert Advisory Group Subgroup on Diet and Health as part of the WHO guideline development process.

For the outcomes with available evidence from randomised controlled trials, additional evidence from non-randomised studies and observational studies can be found in the supplementary materials (supplementary file 1, table 1). If case-control studies and cross sectional studies provided the best available body of evidence, we presented this evidence in the main text. Presentation of the results in this systematic review is primarily structured according to age group (adults or children) and outcome. Within the each outcome, we presented the results for each PICO question separately (that is, population, intervention, comparator, and outcome), describing results of randomised controlled trials first, followed by those of non-randomised and observational studies.

\section{Patient and public involvement}

No patients were involved in setting the research question or the outcome measures, nor were they involved in developing plans for design or implementation of this systematic review. No patients were asked to advise on interpretation or writing up of results. The results of this review will be disseminated to appropriate audiences. It was not evaluated whether the studies included in the review had any patient involvement.

\section{Results}

Details of the study selection are presented in figure 2 . Key characteristics of all included studies are available in supplementary file 3.

Detailed results of the assessment of risk of bias in included randomised controlled trials $(n=21)$ are summarised in supplementary file 1. Unclear reporting about random sequence generation and allocation concealment were the main reasons for unclear risk of bias in randomised controlled trials, while lack of blinding of participants and personnel was the main reason for high risk of bias. Other potential sources of bias were rarely suspected. The overall risk of bias assessment of controlled clinical trials and observational studies $(n=35)$ was serious mainly due to suspected bias caused by confounding, and bias caused by classification of the intervention. The risk of bias assessment for individual non-randomised studies can be found in supplementary file 2 .

NSS intake and health outcomes in adults We included 17 randomised controlled trials, ${ }^{18} 29$ 30-44 six controlled clinical trials, ${ }^{45-49}$ five prospective or retrospective cohort studies, ${ }^{50-54} 15$ case-control 


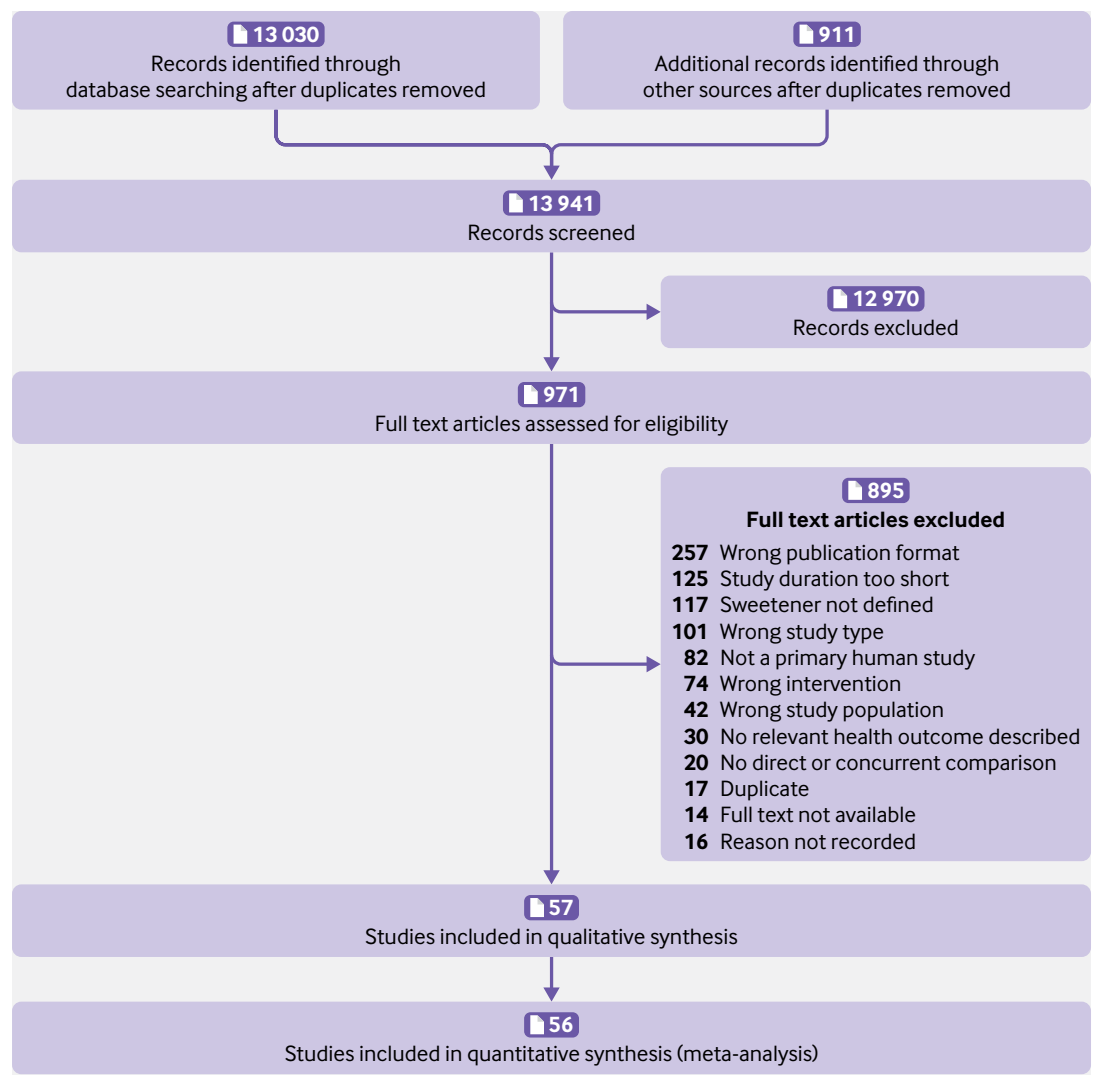

Fig 2 | PRISMA (preferred reporting items for systematic reviews and meta-analyses) flowchart of included studies ${ }^{28}$

studies, ${ }^{55-69}$ and five cross sectional studies ${ }^{70-74}$ in our assessment of the association between health outcomes and NSS intake in adults. We identified seven ongoing studies in adults ${ }^{75-81}$ and one study awaiting classification..$^{82}$

\section{Body weight}

In randomised controlled trials, we saw no significant differences in change in body weight between adults receiving NSSs compared with those receiving different sugars or placebo (mean difference $1.29 \mathrm{~kg}$, 95\% confidence interval -2.80 to 0.21 ; five studies, $n=229$, very low certainty of evidence; fig 3 ). Only one study used placebo as a comparator ${ }^{38}$ while the other studies used caloric sweeteners as a comparator. ${ }^{34} 373940$ There seemed to be no consistent difference in effect between studies using aspartame, ${ }^{343740}$ stevia, $^{38}$ or a combination of sweeteners ${ }^{39}$ as the intervention.

Subgroup analysis by body weight status suggested that NSS use by overweight or obese individuals (that is, those not trying to lose weight, mean body weight $86.87 \mathrm{~kg}$ ) resulted in reduced body weight of 1.99 $\mathrm{kg}$ (95\% confidence interval -2.84 to -1.14 ; three studies, $n=146$, duration of studies, four weeks to six months) but no change in individuals of normal weight (0.03 kg, -0.03 to 0.09; two, $n=110$; fig 3). As assessed in randomised controlled trials, change in body mass index was 0.6 units lower in adults receiving NSSs than in those receiving sucrose $(95 \%$ confidence interval -1.19 to -0.01 ; two studies, $n=174$, low certainty of evidence). Otherwise, randomised controlled trials, non-randomised controlled trials, and observational studies comparing NSS use with no use and with insufficient data for a meta-analysis indicated no consistent difference between the intervention and control group in relation to difference in body weight and other measures of overweight and obesity (supplementary material file 1, table 1).

In one cohort study, ${ }^{50}$ researchers assessed different levels of NSS intake and reported that weight gain was $0.09 \mathrm{~kg}$ lower in women consuming up to $5.8 \mathrm{~g}$ saccharin per day compared with women consuming more than $5.8 \mathrm{~g}$ saccharin per day (95\% confidence interval -0.13 to -0.05 ; one study, outcome assessed in $n=17934$, very low certainty of evidence). Two randomised controlled trials ${ }^{3132}$ investigated the effect of NSS intake in overweight populations trying to lose weight, although they did not provide enough data to conduct meta-analysis (standard error or standard deviation not reported). One study ${ }^{31}$ showed no difference in body weight between the study groups (mean difference $0.10 \mathrm{~kg}, 95 \%$ confidence interval -0.31 to $0.11 ; n=163$, low certainty of evidence). The other study ${ }^{32}$ showed no significant differences between the study groups with regard to reduction in body weight, body mass index, or body fat.

\section{Diabetes or glycaemic control}

In two randomised controlled trials, levels of fasting blood glucose were $0.16 \mathrm{mmol} / \mathrm{L}$ lower in the groups receiving aspartame or a combination of NSSs than in groups receiving sugar (95\% confidence interval -0.26 to -0.06 ; two studies, $n=52$, very low certainty of evidence). ${ }^{37}{ }^{39}$ However, no differences were observed in plasma insulin levels (mean difference $-1.60 \mathrm{pmol} / \mathrm{L}, 95 \%$ confidence interval -8.39 to 5.19 ; two, $n=52$ ) or in insulin resistance and $\beta$ cell function as measured by the homoeostatic model assessment of insulin resistance (HOMA-IR; $-0.14,-0.38$ to 0.10 ; two, $n=66$, very low certainty of evidence). ${ }^{37} 39$ Additional markers for diabetes were reported by single studies only (supplementary material file 1 , table 2 ).

\section{Eating behaviour}

Energy intake and appetite-Pooled data from four randomised controlled trials ${ }^{1839-41}(\mathrm{n}=318$ at baseline) showed that mean daily energy intake was $1064.73 \mathrm{~kJ}$ lower in people receiving NSSs than in those receiving sugar (95\% confidence interval -1867.03 to -262.44 ; four studies, $n=278$, very low certainty of evidence; fig 4). Subgroup analysis by study duration and type of sweetener used as the intervention indicated that this result was largely being driven by one study that lasted for 10 weeks and used a combination of aspartame, cyclamate, acesulfame $\mathrm{K}$, and saccharin (mean difference -2597.00, 95\% confidence interval -3125.35 to $-2068.65 ; n=42)$. Studies of short duration (lasting four weeks) using aspartame as the intervention did not show a significant reduction (-598.94, 95\% confidence interval -1445.24 to 247.36; three studies, $n=276$ ). In one randomised 


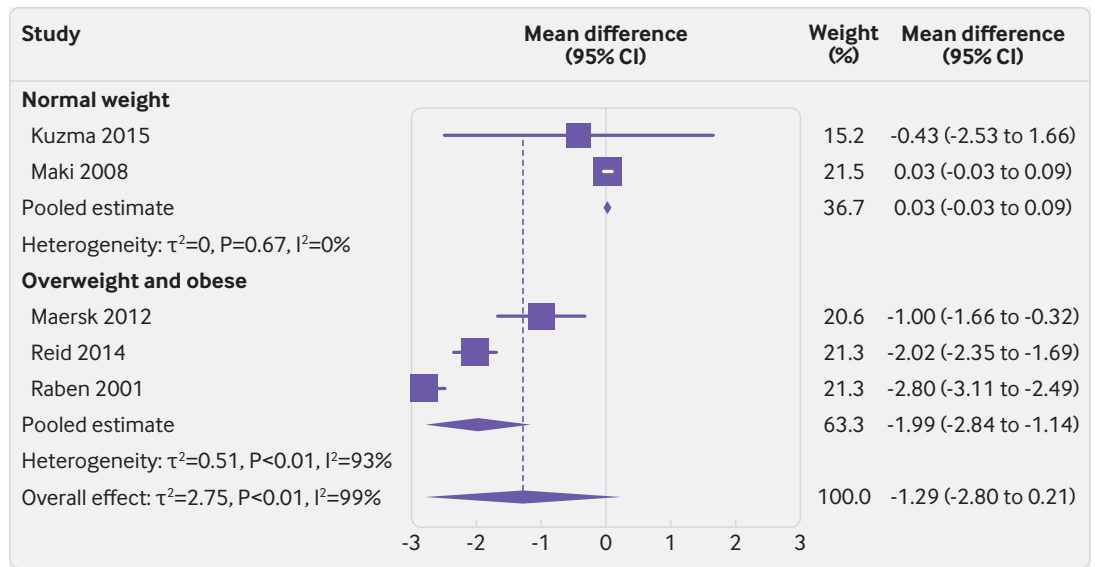

Fig 3 | Effect of non-sugar sweetener intake on weight change $(\mathrm{kg})$ in adults

controlled trial, ${ }^{38}$ researchers reported narratively (that is, without numerical data) that there were no significant differences in energy intake between the stevia and placebo groups. Data from two nonrandomised controlled trials ${ }^{45} 46(n=22)$ suggested no difference between the intervention and control groups for energy intake.

One randomised controlled trial ${ }^{29}$ investigated the effect of NSSs on energy intake in overweight populations trying to lose weight. In this study, mean daily energy intake was reported to be $548 \mathrm{~kJ}$ lower in the group receiving NSSs than in the group avoiding NSSs (95\% confidence interval -692.73 to -403.27 ; $\mathrm{n}=128$ ). In addition, no significant differences were observed for self control with respect to eating (mean difference $-0.20,95 \%$ confidence interval -1.03 to $0.63 ; n=186$, low certainty of evidence) or feelings of hunger $(-0.20,-1.03$ to $0.63 ; n=186$, low certainty of evidence). In another randomised controlled trial, ${ }^{30}$ researchers reported narratively that self reported appetite remained the same in groups receiving NSSs as well as those receiving no intervention over the study period of 12 weeks.

Sugar intake and sweet preference-The pooled effect from three randomised controlled trials ${ }^{18} 3940$ showed that daily sugar intake was $89.71 \mathrm{~g}$ lower in adults receiving NSSs than in those receiving sugar (95\% confidence interval -127.63 to -51.80 ; three studies, $n=135$, very low certainty of evidence; fig 5). All three studies included overweight or obese participants. Both studies by Reid $^{18} 40$ measured sugar intake by including the sucrose from the control intervention in their outcome measure. Data from two non-randomised controlled trials ${ }^{4649}$ and one cross sectional study ${ }^{72}$ showed no differences in sugar intake between the intervention and control groups.

Two randomised controlled trials ${ }^{31} 32$ investigated the effect of NSSs on preference for sweet taste or sugar intake in overweight populations trying to lose weight. The preference for sweet taste, as assessed by desire for sweets (measured on a 0-10 scale with higher values indicating increased desire), was slightly lower in the group receiving NSSs than in the group not receiving NSSs (mean difference $-0.2,95 \%$ confidence interval
-0.34 to -0.06 ; one study, $\mathrm{n}=186$, moderate certainty of evidence). Sugar intake was similar between the groups after three years of follow-up $(-0.00 \mathrm{~g},-0.18$ to 0.18; one, $\mathrm{n}=186$ ). ${ }^{31}$

\section{Cancer}

The risk for bladder or lower urinary tract cancer as assessed in meta-analysis of case control studies seemed to be similar in those exposed to sweeteners and those unexposed to sweeteners (odds ratio 1.03, 95\% confidence interval 0.84 to 1.25 ; eight studies, $\mathrm{n}=4509$, very low certainty of evidence; fig 6 ). The odds ratios for other types of cancer as reported in various observational studies suggested no difference in risk for different cancers except for ovarian cancer ( $0.61,0.38$ to 0.98 ; one case-control study, $n=459$ ) and pancreatic cancer $(0.19,0.08$ to 0.46 , one casecontrol study, $n=978$ ). The certainty of evidence for the risk of different types of cancers was very low.

We saw no association between consumption of higher doses of aspartame and incidence of the main subtypes of lymphoid cancers, non-Hodgkin lymphoma subtypes $(\mathrm{P}=0.69)$, or non-lymphoid leukaemia, in two prospective cohort studies with up to 10 years of follow-up $(\mathrm{n}=473984) .{ }^{5253}$ Similarly, no association was seen between consumption of higher NSS doses and lower urinary tract cancer $(n=149$, very low certainty of evidence) in one case-control study ${ }^{6} 6$

\section{Blood pressure}

Data from three randomised controlled trials showed that systolic and diastolic blood pressure were lower in people receiving NSSs than in those receiving sugar or placebo (systolic, mean difference $-4.90 \mathrm{~mm} \mathrm{Hg}$, $95 \%$ confidence interval -9.78 to -0.03 ; diastolic, $-3.27 \mathrm{~mm} \mathrm{Hg},-7.21$ to 0.67 ; three studies, $\mathrm{n}=202$ at baseline, very low certainty of evidence). ${ }^{37-39}$ The effect seemed stronger in studies using caloric sweeteners as comparators ${ }^{37} 39$ than in those that used a non-caloric comparator. ${ }^{38}$ In another randomised controlled trial, researchers reported narratively that there was no change in blood pressure in the study groups. ${ }^{29}$

No significant differences in systolic and diastolic blood pressure were reported in one randomised controlled trial assessing the effect of aspartame in overweight populations trying to lose weight. ${ }^{32}$ After 12 weeks, the group differences in diastolic blood pressure were $6 \mathrm{~mm} \mathrm{Hg}$ less in men and $1 \mathrm{~mm} \mathrm{Hg}$ more in women when the aspartame group was compared with controls (not enough data for formal statistical comparison, very low certainty of evidence).

\section{Other outcomes}

In studies comparing NSS intake with no intake, we found an increased risk of depression in one cohortstudy (odds ratio 1.14, 95\% confidence interval 1.02 to 1.27; $\mathrm{n}=263$ 923). ${ }^{51}$ We also found no effects on the incidence of kidney disease (very low certainty of evidence), ${ }^{44}$ mood (moderate certainty of evidence), ${ }^{18} 294042$ behaviour (very low certainty of evidence), ${ }^{83}$ neurocognition (low certainty of evidence), ${ }^{42}$ or risk of 
adverse events (eg, skin reactions, loss of appetite, and headaches; risk ratio $0.65,95 \%$ confidence interval 0.16 to 2.59 ; three studies, $\mathrm{n}=167$, low certainty of evidence). ${ }^{294244}$ We identified no studies investigating the incidence of asthma or the incidence of allergies.

In studies comparing different doses of NSS intake, evidence from one crossover randomised controlled trial $^{36}$ indicated a significant increase in depression in people consuming the higher aspartame dose compared with those consuming the lower dose (low certainty of evidence). The study reported significantly better results in participants receiving lower doses of aspartame with respect to neurocognition (low certainty of evidence), but no difference in adverse events for higher intake versus lower intake of aspartame (low certainty of evidence). ${ }^{36}$ Similarly, in two randomised controlled trials, ${ }^{31}{ }^{32}$ no significant differences in the risk for adverse events were observed between individuals receiving NSSs and those not receiving NSSs in overweight populations trying to lose weight (risk ratio 1.38, 95\% confidence interval 0.58 to $3.28 ; n=204$, low certainty of evidence). Detailed results on all outcomes are reported in supplementary file 1.

NSS intake and health outcomes in children

Overall, we identified four randomised controlled trials, ${ }^{84-87}$ two non-randomised controlled trials, ${ }^{83} 88$ one case-control study, ${ }^{89}$ and one cross sectional study ${ }^{70}$ that contributed data to our review regarding the association between NSS intake and health outcomes in children. We identified one ongoing study in children. ${ }^{90}$

\section{Body weight}

Two randomised controlled trials ${ }^{85} 91$ found a similar weight gain in children receiving sucralose and acesulfame $\mathrm{K}^{91}$ or aspartame ${ }^{85}$ and children receiving sucrose (mean difference $-0.60 \mathrm{~kg}, 95 \%$ confidence interval -1.33 to 0.14 ; two studies, $n=467$, low certainty of evidence; fig 7). After exclusion of the oldest age group (13-21 years) from one study ${ }^{85}$ in a sensitivity analysis, we saw no difference in effect $(-0.50 \mathrm{~kg},-1.43$ to 0.42 ; two, $\mathrm{n}=722)$. Two randomised controlled trials ${ }^{87} 92$ reported a significantly smaller increase in body mass index $\mathrm{z}$ score in children receiving sucralose and acesulfame $\mathrm{K}^{91}$ or sucralose alone, ${ }^{87}$ compared with children receiving sucrose $(-0.15,-0.17$ to $-0.12 ; n=528$, moderate certainty of evidence).

One randomised controlled trial ${ }^{92} \quad(n=641)$ reported no group differences in body fat measured by electrical impedance (mean difference $-0.83 \%$ body fat, 95\% confidence interval $-2.12 \%$ to $0.46 \%$ ), waist circumference $(-0.50 \mathrm{~cm},-1.73$ to 0.73$)$, skinfold thickness $(-1.5 \mathrm{~mm},-4.71$ to 1.71$)$, and waist-to-height ratio $(-0.50 \%,-1.73$ to 0.73$)$. In one randomised controlled trial including overweight or obese children involved in a weight loss programme, ${ }^{86}$ researchers reported a lower weight gain in children receiving aspartame than in children receiving placebo
$(-0.75 \mathrm{~kg},-1.08$ to -0.43 ; one study, $\mathrm{n}=57$, low certainty of evidence).

\section{Dental health}

In one non-randomised controlled trial, ${ }^{88}$ mouth rinses with chlorhexidine were more effective than stevioside in decreasing plaque volume. Plaque volume was similar in the groups using water or stevioside (low certainty of evidence).

\section{Eating behaviour}

Satiety, appetite, and energyintake-In one randomised controlled trial $(n=141)$, children receiving NSSs versus those receiving sucrose had similar self reported satiety one minute after intake (odds ratio 0.77, 95\% confidence interval 0.46 to 1.29 ) and 15 minutes after intake (1.44, 0.86 to 2.40$).{ }^{84}$ Self reported appetite increase (risk ratio $0.84,95 \%$ confidence interval 0.22 to 3.29 ) or appetite decrease (1.08, 0.44 to 2.63 ) were similar between the study groups in another randomised controlled trial $(n=126) .{ }^{85}$ According to evidence from a third randomised controlled trial, energy intake was lower in the sucralose group than in the sucrose group (mean difference $197.60 \mathrm{~kJ}$, $95 \%$ confidence interval -327.18 to 722.38 ; $n=190$, low certainty of evidence). ${ }^{87}$ In one non-randomised controlled trial, mean daily energy intake was reported to be similar between the groups receiving aspartame or saccharin and significantly increased in the group that received sucrose. ${ }^{83}$ Energy intake was 6711, 6640, or $7728 \mathrm{~kJ}$ daily with aspartame, saccharin, or sucrose in the preschool group, respectively, and 8100, 8284, and $9293 \mathrm{~kJ}$ for school age children, respectively. In one randomised controlled trial with overweight children involved in active weight loss, researchers assessed change in appetite as self reported adverse events, which were reported to be no different between the study groups (incidence rate ratio 0.94, 95\% confidence interval 0.35 to 2.49 ; one study, $n=55$, very low certainty of evidence). ${ }^{86}$

Preference for sweet taste-One crossover nonrandomised controlled trial $^{83} \quad(\mathrm{n}=47)$ reported significantly lower sugar intake in children receiving aspartame or saccharin than in children receiving sucrose (not enough data for formal statistical comparison, very low certainty of evidence). The effect seemed to be strongly related to the sugar content of the experimental diets.

\section{Diabetes}

In one crossover non-randomised controlled trial, ${ }^{83}$ researchers found a significantly higher increase in blood glucose in children of preschool age receiving aspartame compared with sucrose (mean difference $0.24 \mathrm{mmol} / \mathrm{L}, 95 \%$ confidence interval 0.09 to 0.39 ; $\mathrm{n}=25$ ), a significantly higher increase in blood glucose in children of school age receiving saccharin compared with sucrose ( $0.65 \mathrm{mmol} / \mathrm{L}, 0.44$ to $0.86 ; \mathrm{n}=23)$, and a significantly lower increase in blood glucose in children of preschool age receiving aspartame compared with saccharin $(-0.75 \mathrm{mmol} / \mathrm{L},-0.95$ to $-0.64 ; \mathrm{n}=23$, very 


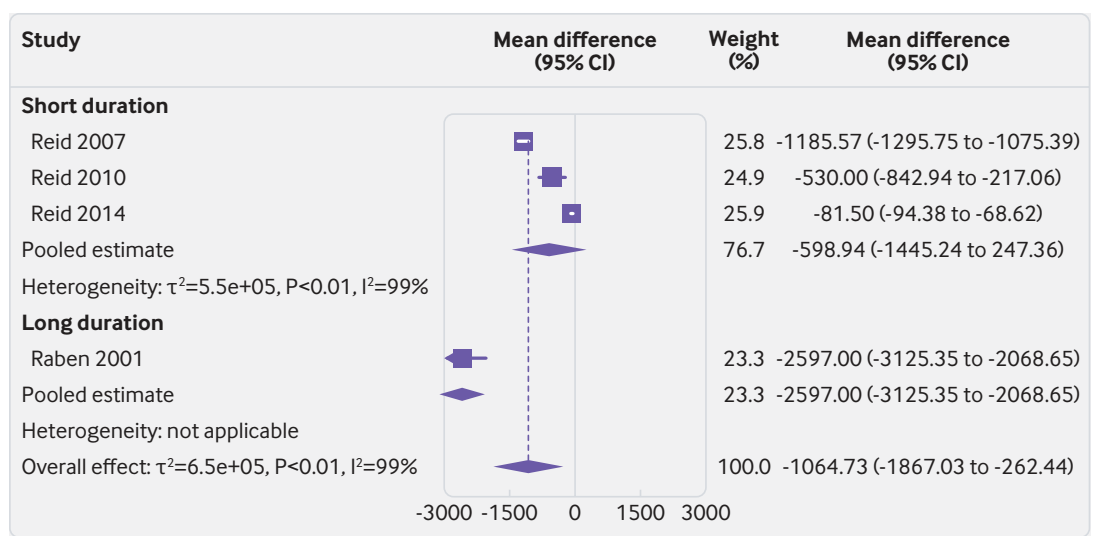

Fig 4 | Effect of non-sugar sweetener intake on daily energy intake (kJ) by study duration (4 or 10 weeks)

low certainty of evidence). In overweight children involved in active weight loss, blood glucose decreased less strongly in those receiving NSSs compared with those not receiving NSSs $(0.3 \mathrm{mmol} / \mathrm{L}, 0.2$ to 0.4 ; $\mathrm{n}=49$, very low certainty of evidence). ${ }^{86}$

\section{Cancer}

In one case-control study ${ }^{89}(\mathrm{n}=150)$, researchers reported no difference in risk for primary brain tumours when looking at aspartame intake from all sources (risk ratio 1.1, 95\% confidence interval 0.5 to 2.6) or aspartame intake from diet drinks only $(0.9,0.3$ to 2.4; very low certainty of evidence). Furthermore, no difference in risk of primary brain tumours was seen with different durations or frequencies of aspartame intake (very low certainty of evidence; see supplementary material file 1 , table 4).

\section{Cardiovascular disease}

In one randomised controlled trial, ${ }^{85}$ total cholesterol concentration decreased strongly in sucrose groups but increased in the aspartame group (mean difference $0.44 \mathrm{mmol} / \mathrm{L}, 95 \%$ confidence interval 0.33 to 0.56 ; $\mathrm{n}=45)$. The change in triglyceride concentration (4.00, -0.50 to $8.50 ; n=45$, unit of measurement not reported) and blood pressure (no numerical data reported, very low certainty of evidence) were similar between the study groups. Another randomised controlled trial ${ }^{86}$ reported that in overweight children involved in active weight loss, systolic and diastolic blood pressure were similar in those receiving NSSs or placebo (systolic,

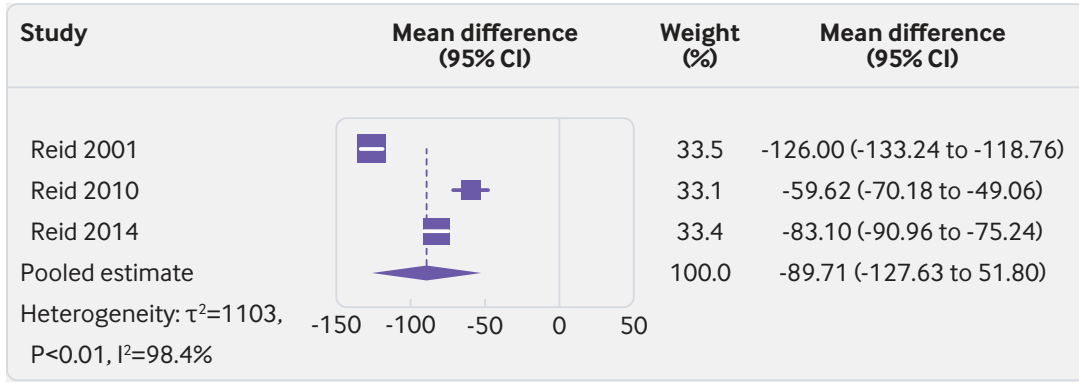

Fig 5 | Effect of non-sugar sweetener intake on daily sugar intake (g) in adults mean difference $1.00 \mathrm{~mm} \mathrm{Hg}, 95 \%$ confidence interval -0.95 to 2.95; diastolic, $1.00 \mathrm{~mm} \mathrm{Hg},-0.53$ to 2.53 ; $\mathrm{n}=55$, very low certainty of evidence).

\section{Kidney disease}

In randomised controlled trials, no differences were observed in concentrations of creatinine (an intermediate marker for kidney disease) between NSS intake and no intake in overweight children involved in weight loss studies (mean difference 0.002 $\mathrm{mmol} / \mathrm{L}, 95 \%$ confidence interval -0.001 to 0.005 ; one, $n=49$, very low certainty of evidence). ${ }^{86}$ Similarly, no corresponding difference was seen in children of healthy weight $(0.003,-0.012$ to 0.018 ; one, $n=126$ at baseline, very low certainty of evidence). ${ }^{85}{ }^{86}$ However, after exclusion of the oldest age group (13-21 years) in a sensitivity analysis, creatinine decreased more strongly in the sucrose group $(0.011,0.004$ to 0.018 ; $\mathrm{n}=80)$.

\section{Other outcomes}

In one non-randomised controlled trial, we found no difference in effect between children receiving NSSs and those not receiving NSSs on self rated mood states (very low certainty of evidence), ${ }^{83}$ behaviour (very low certainty of evidence), ${ }^{83}$ and cognitive performance (low certainty of evidence). ${ }^{83}$ One randomised controlled trial ${ }^{87}$ described significantly worse neurocognitional performance in tests of cognitive abilities in children receiving NSSs than in children receiving sugar $(n=386)$. Another randomised controlled trial reported no difference in the occurrence of adverse events between children receiving NSSs and children not receiving NSSs (risk ratio $1.28,95 \%$ confidence interval 0.86 to 1.91 ; $\mathrm{n}=126$, low certainty of evidence) ${ }^{85}$ However, in one randomised controlled trial, a higher risk of adverse effects in overweight children involved in active weight loss not receiving NSSs versus those receiving NSSs was observed (incidence rate ratio 1.37, 95\% confidence interval 1.05 to $1.79 ; n=55$, low certainty of evidence). ${ }^{86}$ Overall, 103 adverse effects were noted in the intervention group and 113 in the control group. We identified no studies investigating the effect of NSS intake on incidence of asthma or allergies.

\section{Discussion}

\section{Principal findings}

This comprehensive systematic review covers a broad range of benefits and harms of NSSs in a generally healthy population of adults and children, following rigorous systematic review methods. Overall, we included 56 studies of adults and children, which assessed the associations and effects of NSSs on different health outcomes. For most outcomes, there seemed to be no statistically or clinically relevant difference between NSS intake versus no intake, or between different doses of NSSs. No evidence was seen for health benefits from NSSs and potential harms could not be excluded. The certainty of the 


\begin{tabular}{|c|c|c|c|}
\hline Study & $\begin{array}{l}\text { Odds ratio } \\
(95 \% \mathrm{Cl})\end{array}$ & $\begin{array}{l}\text { Weight } \\
\text { (\%) }\end{array}$ & $\begin{array}{l}\text { Odds ratio } \\
(95 \% \mathrm{Cl})\end{array}$ \\
\hline Simon 1975 & & 10.2 & $1.00(0.59$ to 1.70$)$ \\
\hline Kessler 1978 & & 17.3 & $0.88(0.62$ to 1.25$)$ \\
\hline Cartwright 1981 & & 23.1 & 1.20 (0.93 to 1.55$)$ \\
\hline Najem 1982 & & 5.4 & 1.25 (0.57 to 2.75$)$ \\
\hline Möller-Jensen (Saccharin) 1983 & & 17.3 & $0.76(0.53$ to 1.07$)$ \\
\hline Möller-Jensen (Cyclamate) 1983 & & 4.3 & 0.84 (0.34 to 2.07$)$ \\
\hline Mommsen 1983 & & 1.4 & 6.73 (1.30 to 34.78$)$ \\
\hline Nomura 1991 & & 14.3 & $0.96(0.64$ to 1.46$)$ \\
\hline Momas 1994 & & 6.7 & $1.50(0.75$ to 3.00$)$ \\
\hline Pooled estimate & & 100.0 & $1.03(0.84$ to 1.25$)$ \\
\hline \multicolumn{4}{|l|}{ Heterogeneity: $\tau^{2}=0.027, P=0.16, I^{2}=32 \%$} \\
\hline 0.1 & 0.5 & 10 & \\
\hline
\end{tabular}

Fig 6 | Effect of non-sugar sweetener intake on risk (odds ratio) of bladder cancer. Odds ratio of less than $1=$ increased risk of cancer with non-sugar sweeteners

included evidence ranged from very low to moderate, and our confidence in the reported effect estimates is accordingly limited.

\section{Strengths and weaknesses in relation to other studies}

In a preparatory mapping review, ${ }^{93}$ we identified 372 primary and secondary studies that investigated the effects of NSS intake on different health outcomes. However, the methodological and reporting quality of many publications was limited. Most studies did not contain enough information on the study design or lacked other reporting detail-that is, the sweetener used was not transparently reported, such that many the studies identified in the mapping review were not eligible for this systematic review. Studies included in this systematic review were rarely comparable with regard to their aim, design, and methods so that meaningful comparisons between them was challenging.

Although most studies reported sufficient detail for the population included, few reported sufficient information on the intervention, comparator, and outcomes. For example, comparisons of effects of different doses of sweeteners in children were not possible because most studies did not report the respective information on dose. Additionally, reported doses and outcomes measures were reported so differently that we could not assess the effect of dose on any outcome (eg, two studies ${ }^{83}{ }^{85}$ reported dose of aspartame and assessed eating behaviour, but the outcome was measured as energy intake or as a decrease in appetite). Furthermore, outcomes of relevance for

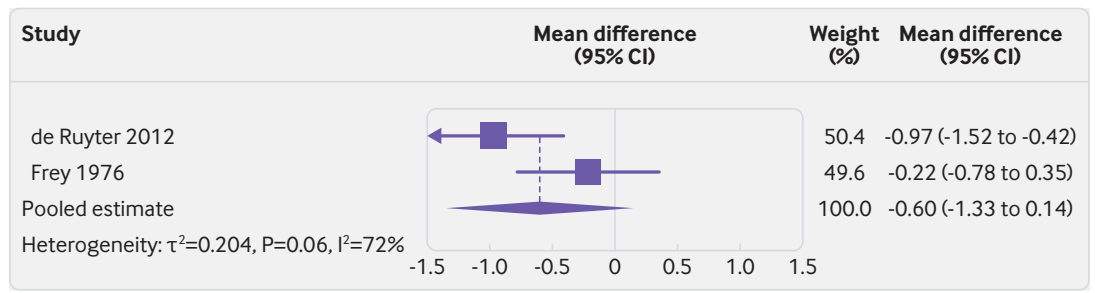

Fig 7 | Effect of non-sugar sweetener intake on risk (odds ratio) of bladder cancer. Odds ratio of less than $1=$ increased risk of cancer with non-sugar sweeteners this review were often only measured indirectly with intermediate markers. Lastly, most included studies had small sample sizes and their study duration was often too short to infer any meaningful results in the longer term.

Several other systematic and narrative reviews have examined the effects of NSSs on various health outcomes. $^{345694-97}$ The methodological and clinical inclusion and exclusion criteria used in these systematic reviews differed substantially from our criteria in the present study, resulting in a different pool of included studies. The data synthesis methods also differed from the ones used in the present review. Still, the reviews found similar results to our results: Brown and colleagues ${ }^{4}$ found no strong clinical evidence for an effect of artificial sweeteners on metabolic effect in youths, whereas Cheungpasitporn and colleagues ${ }^{3}$ found no effect of artificially sweetened soda on chronic kidney disease. Greenwood and colleagues ${ }^{5}$ reported no consistent association between artificially sweetened soft drinks and diabetes risk. Onakpoya and Henegham ${ }^{95}$ reported a non-significant reduction in systolic blood pressure and significant reductions in diastolic blood pressure and fasting blood glucose with steviol glycoside compared with placebo, but indicated that the evidence was not robust due to heterogeneity.

Wiebe and colleagues ${ }^{6}$ reported a decrease in body mass index in people consuming foods and drinks containing non-caloric sweeteners compared with an increased body mass index in those consuming foods and drinks containing sucrose. The researchers further highlighted the lack of high quality research regarding non-caloric sweeteners. A systematic review by Azad and colleagues ${ }^{97}$ found no statistically significant effect of non-nutritive sweeteners on body mass index, body weight, fat mass, waist circumference, and HOMA-IR. Overall, published systematic reviews rarely drew firm conclusions. Main methodological concerns were limitations in the literature search and the data analyses. By contrast to our review, most meta-analyses were not planned and conducted, and the authors summarised the individual study results narratively instead.

A few large prospective cohort studies ${ }^{98-102}$ with long term follow-up investigated the association between NSS intake and different health outcomes. However, the NSSs being investigated were not sufficiently specified to match the inclusion criteria of this review. Still, their results indicate an increased risk of higher body mass index and type 2 diabetes with higher NSS consumption, or lower risk of cardiovascular disease with intake of artificially sweetened sodas compared with sugar sweetened sodas. These results partly conflict with the ones from the findings of this systematic review. Included studies investigated long term health outcomes for a relatively short duration - for example, cardiovascular health ${ }^{29} 3337-3944474871728586$ outcomes or diabetes 35373944728386 investigated for six months or less. Long term studies with sufficient statistical power are key to investigating long term health outcomes such as incidence of diabetes or 
cardiovascular health. Hence, results of large, long term cohort studies should be verified by studies that specify the type of sweeter used.

The findings of our review might be biased by the fact that only one reviewer assessed inclusion of studies in the initial title and abstract screening phase. Hence, relevant references could have inadvertently not been included in this review. However, this possibility is unlikely because only clearly irrelevant references were excluded at this stage. Furthermore, we did not seek clarification with the study authors about whether our assessment of risk of bias in the individual studies was correct. In the statistical analyses, missing standard deviations for change in outcomes were imputed, and in some cases, approximation was used for the analyses. ${ }^{103}$ Therefore, the reliability of analyses of changes in outcomes might have been weakened by the unavailability of data and the use of imputed values and approximation.

Implications for clinicians and policy makers, unanswered questions, and future research

This review was prepared to inform a WHO guideline on NSS use. The guideline will provide information on implications for actions by health experts and policy makers. So far, several studies on the effects of NSSs on different health outcomes have been conducted. However, their methodological or reporting quality is mostly limited and often not sufficiently detailed to include their results in meta-analyses. Moreover, included studies differed substantially in their design (that is, choice of population, intervention, comparator, and outcome measures). Given these relevant differences between studies, a reliable review of the effects by type of sweetener or of the caloric effects versus non-caloric effects is challenging. Type of intervention and comparator might affect health outcomes differently and should be considered in future research.

We also recommend that future studies assess the effects of NSS use on health outcomes with an appropriate study duration. Study planning should consider the duration necessary for plausible, relevant effects to occur in the different outcomes of interest. Longer term studies are needed to assess effects on overweight and obesity, risk for diabetes, cardiovascular disease, and kidney disease. Type and dose of sweetener use should be reported precisely and transparently in all studies. Precise reporting of sweetener content (that is, type and amount of sweeteners) in ready-to-consume foods and beverages is highly desirable and could be helped by more detailed information on ingredients as provided by manufacturers. Consistent use of core outcome measures and consensus on timing and mode of assessment would further help researchers pool data across studies. In addition to studying the effects on NSS use in a general healthy population of adults and children, research should focus on diseased populations and other subgroups, including pregnant women and their offspring and people who use NSSs in amounts higher than average (such as those with diabetes). ${ }^{104}$

Most of the studies identified for this review used single sweeteners and the use patterns of sweeteners in the studies might differ from that in real life practice. ${ }^{105}$ Therefore, the certainty in the evidence presented in this review might further be affected by indirectness. For example, NSSs can be consumed in different ways, including as a table top sweetener (that is, added to tea or coffee as a replacement for sugar) where the dose is freely determined by users themselves and might be higher than in that recorded the studies. Moreover, by contrast to many of our included studies that used a single NSS only, many food items have different types of NSSs that are combined to cover different bitter or metallic aftertastes of individual sweeteners and provide an adequate sweetness. Future research might consider exploring the effects of different combinations of sweeteners in doses similar to real life use patterns and compare the effects of higher versus lower NSS doses. Development and research on NSSs is ongoing, and new alternatives to sugar are presented on a regular basis. Therefore, we also need data on the safety and benefits and harms of other sweeteners not assessed in this review for a comprehensive overview of the health effects of NSSs.

Results of observational studies on the health effects of NSSs should be interpreted with caution, and attention should focus on plausible residual confounding as well as reverse causality (such as a higher consumption of NSSs by overweight or obese populations aiming at weight management). ${ }^{106}$ Appropriate long term studies that consider baseline consumption of sugar and $\mathrm{NSSs}^{105}$ and have an appropriate comparator ${ }^{106}$ should investigate whether NSSs are a safe and effective alternative to sugar, and results should be interpreted in light of these study design characteristics. ${ }^{105} 106$

The WHO Nutrition Guidance Advisory Group (NUGAG) Subgroup on Diet and Health provided valuable insight on aims and objectives of this review. WHO agreed to the publication of this systematic review in a scientific journal because it serves as the background evidence review for WHO guidelines on non-sugar sweeteners and should therefore be available widely.

Contributors: SL, IT, and JJM conceived and designed the review. JJM coordinated the review. SL, JJM, and IT designed the search strategy. SL and IT undertook the searches and screened the search results. IT organised the retrieval of the papers. SL, IT, DKdG, and JJM screened the papers against eligibility criteria. SL, IT, and DKdG appraised the quality of the papers. SL, IT, DKdG, and HS extracted data from the papers. HS analysed the data. SL, JJM, IT, and DKdG interpreted data. IT, SL, DKdG, and JJM wrote the review and its protocol. JJM, SL, and DKdG provided general advice on the review. JJM secured funding for the review. SL, JJM, and IT performed previous work that was the foundation of the current review. JJM is the guarantor of this manuscript. The questions guiding the review were discussed and developed by the WHO NUGAG Subgroup on Diet and Health, and the study protocol was approved, by the NUGAG Subgroup on Diet and Health. Neither WHO nor the WHO NUGAG Subgroup on Diet and Health played a role in data collection or analysis. All authors, external and internal, had full access to all of the data (including statistical reports and tables) in the study and can take responsibility for the integrity of the data and the accuracy of the data analysis. The corresponding author attests that all listed authors meet authorship criteria and that no others meeting the criteria have been omitted. 
Funding: The research was funded by WHO. The research was conducted independently from the funder, and researchers are independent from the funder.

Competing interests: All authors have completed the ICMJE uniform disclosure form at www.icmje.org/coi_disclosure.pdf and declare: support from WHO for the submitted work; no financial relationships with any organisations that might have an interest in the submitted work in the previous three years; no other relationships or activities that could appear to have influenced the submitted work.

Ethical approval: Ethical approval was not required for this research.

Data sharing: Full datasets can be obtained from the corresponding author at Meerpohl@cochrane.de.

The lead author affirms that the manuscript is an honest, accurate, and transparent account of the study being reported; that no important aspects of the study have been omitted; and that any discrepancies from the study as planned (and, if relevant, registered) have been explained.

This is an Open Access article distributed in accordance with the Creative Commons Attribution Non Commercial (CC BY-NC 4.0) license, which permits others to distribute, remix, adapt, build upon this work non-commercially, and license their derivative works on different terms, provided the original work is properly cited and the use is noncommercial. See: http://creativecommons.org/licenses/by-nc/4.0/.

1 Sakurai M, Nakamura K, Miura K, et al. Sugar-sweetened beverage and diet soda consumption and the 7-year risk for type 2 diabetes mellitus in middle-aged Japanese men. Eur J Nutr 2014;53:251-8. doi:10.1007/s00394-013-0523-9

2 Miller PE, Perez V. Low-calorie sweeteners and body weight and composition: a meta-analysis of randomized controlled trials and prospective cohort studies. Am J Clin Nutr 2014;100:765-77. doi:10.3945/ajcn.113.082826

3 Cheungpasitporn W, Thongprayoon C, O'Corragain OA, Edmonds PJ, Kittanamongkolchai W, Erickson SB. Associations of sugarsweetened and artificially sweetened soda with chronic kidney disease: a systematic review and meta-analysis. Nephrology (Carlton) 2014;19:791-7. doi:10.1111/nep.12343

4 Brown RJ, de Banate MA, Rother KI. Artificial sweeteners: a systematic review of metabolic effects in youth. Int J Pediatr Obes 2010;5:30512. doi:10.3109/17477160903497027

5 Greenwood DC, Threapleton DE, Evans CE, et al. Association between sugar-sweetened and artificially sweetened soft drinks and type 2 diabetes: systematic review and dose-response meta-analysis of prospective studies. Br J Nutr 2014;112:725-34. doi:10.1017/ S0007114514001329

6 Wiebe N, Padwal R, Field C, Marks S, Jacobs R, Tonelli M. A systematic review on the effect of sweeteners on glycemic response and clinically relevant outcomes. BMC Med 2011;9:123. doi:10.1186/1741-7015-9-123

7 Olivier B, Serge AH, Catherine A, et al. Review of the nutritional benefits and risks related to intense sweeteners [correction in: Arch Public Health 2015:73:49]. Arch Public Health 2015:73:41.

8 Wong DWS. Mechanism and theory in food chemistry. 2nd ed. Sweeteners. Springer, 2018: 309-25.

9 Sardarodiyan M, Hakimzadeh V. Artificial sweeteners. Int J Pharm Tech Res 2016;9:357-63.

10 Ferrazzano GF, Cantile T, Alcidi B, et al. Is Stevia rebaudiana Bertoni a Non Cariogenic Sweetener? A Review. Molecules 2015;21:E38. doi:10.3390/molecules21010038

11 Mortensen A. Sweeteners permitted in the European Union: safety aspects. Food Nutr Res 2006;50:104-16 doi:10.1080/17482970600982719.

12 US Food and Drug Administration. Additional information about high-intensity sweeteners permitted for use in food in the United States: US Food and Drug Administration; 2017. https://www.fda. gov/food/ingredientspackaginglabeling/foodadditivesingredients/ ucm 397725.htm

13 Authority EFS. Sweeteners Brussels, Belgium. 2017. http://www.efsa. europa.eu/en/topics/topic/sweeteners.

14 EFSA Panel on Dietetic Products NaA. Scientific opinion on the substantiation of health claims related to intense sweeteners and contribution to the maintenance or achievement of a normal body weight (ID 1136, 1444, 4299), reduction of post-prandial glycaemic responses (ID 4298), maintenance of normal blood glucose concentrations (ID $1221,4298)$, and maintenance of tooth mineralisation by decreasing tooth demineralisation (ID 1134, 1167, 1283) pursuant to Article 13(1) of Regulation (EC) No 1924/2006. Parma, Italy, 2011. https://efsa. onlinelibrary.wiley.com/doi/abs/10.2903/j.efsa.2011.2229

15 World Health Organization. WHO handbook for quideline development. 2nd ed. World Health Organization, 2014
16 Higgins JPT, Green S. Cochrane handbook for systematic reviews of interventions. Version 5.1.0. John Wiley \& Sons, 2011.

17 World Health Organization. Evaluations of the Joint FAO/WHO Expert Committee on Food Additives (JECFA). 2017. http://apps.who.int/ food-additives-contaminants-jecfa-database/search.aspx.

18 Reid M, Hammersley R, Duffy M. Effects of sucrose drinks on macronutrient intake, body weight, and mood state in overweight women over 4 weeks. Appetite 2010;55:130-6. doi:10.1016/j. appet.2010.05.001

19 Ebbeling CB, Feldman HA, Chomitz VR, et al. A randomized trial of sugar-sweetened beverages and adolescent body weight. $N$ Engl Med 2012:367:1407-16. doi:10.1056/NEIMoa1203388

20 European Food Safety Authority. Revised exposure assessment for steviol glycosides for the proposed uses as a food additive. 2016 http://www.efsa.europa.eu/de/efsajournal/pub/1972.

21 Lefebvre C, Manheimer E, Glanville J. Searching for studies. Cochrane Handbook for Systematic Reviews of Interventions. John Wiley \& Sons, 2008: 95-150. doi:10.1002/9780470712184.ch6.

22 Jelicic Kadic A, Vucic K, Dosenovic S, Sapunar D, Puljak L. Extracting data from figures with software was faster, with higher interrater reliability than manual extraction. J Clin Epidemiol 2016;74:119-23. doi:10.1016/j.jclinepi.2016.01.002

23 Nettleton JA, Lutsey PL, Wang Y, Lima JA, Michos ED, Jacobs DRJr. Diet soda intake and risk of incident metabolic syndrome and type 2 diabetes in the Multi-Ethnic Study of Atherosclerosis (MESA). Diabetes Care 2009:32:688-94. doi:10.2337/dc08-1799

24 Sterne JA, Hernán MA, Reeves BC, et al. ROBINS-I: a tool for assessing risk of bias in non-randomised studies of interventions. BMJ 2016;355:i4919. doi:10.1136/bmj.i4919

25 Hozo SP, Djulbegovic B, Hozo I. Estimating the mean and variance from the median, range, and the size of a sample. BMC Med Res Methodol 2005;5:13. doi:10.1186/1471-2288-5-13

26 R Development Core Team. R: A language and environment for statistical computing. R Foundation for Statistical Computing, 2011.

27 Guyatt GH, Oxman AD, Vist GE, et al, GRADE Working Group. GRADE: an emerging consensus on rating quality of evidence and strength of recommendations. BMJ 2008;336:924-6. doi:10.1136/ bmj.39489.470347.AD

28 GRADEpro GDT. GRADEpro guideline development tool. McMaster University, 2015

29 Baird IM, Shephard NW, Merritt RJ, Hildick-Smith G. Repeated dose study of sucralose tolerance in human subjects. Food Chem Toxicol 2000;38(Suppl 2):S123-9. doi:10.1016/S02786915(00)00035-1

30 Ballantyne C), Hammersley R, Reid M. Effects of sucrose added blind to the diet over eight weeks on body mass and mood in men. Appetite 2011;57:S3. doi:10.1016/j.appet.2011.05.118.

31 Blackburn GL, Kanders BS, Lavin PT, Keller SD, Whatley J. The effect of aspartame as part of a multidisciplinary weight-control program on short- and long-term control of body weight. Am J Clin Nutr 1997;65:409-18. doi:10.1093/ajcn/65.2.409

32 Kanders BS, Lavin PT, Kowalchuk MB, Greenberg I, Blackburn GL. An evaluation of the effect of aspartame on weight loss. Appetite 1988;11(Suppl 1):73-84. doi:10.1016/S0195 6663(88)80050-3

$33 \mathrm{Kim}$ EJ, Kim MY, Kim JS, et al. Effects of fructooligosaccharides intake on body weight, lipid profiles, and calcium status among Korean college students. FASEB / 2011;25(Suppl 1)

34 Kuzma JN, Cromer G, Hagman DK, et al. No difference in ad libitum energy intake in healthy men and women consuming beverages sweetened with fructose, glucose, or high-fructose corn syrup: a randomized trial. Am J Clin Nutr 2015;102:1373-80. doi:10.3945/ ajcn.115.116368

35 Lee BH, Kim EJ, Kim MY, et al. Effect of fructooligosaccharides on improvement of blood glucose, calcium status and habitual bowel movement among college students in Korea. FASEB / 2012;26(Suppl 1).

36 Lindseth GN, Coolahan SE, Petros TV, Lindseth PD. Neurobehavioral effects of aspartame consumption. Res Nurs Health 2014;37:18593. doi:10.1002/nur.21595

37 Maersk M, Belza A, Stødkilde-Jørgensen H, et al. Sucrose-sweetened beverages increase fat storage in the liver, muscle, and visceral fat depot: a 6-mo randomized intervention study. Am J Clin Nutr 2012:95:283-9. doi:10.3945/ajcn.111.022533

38 Maki KC, Curry LL, Carakostas MC, et al. The hemodynamic effects of rebaudioside $A$ in healthy adults with normal and low-normal blood pressure. Food Chem Toxicol 2008;46(Suppl 7):S40-6. doi:10.1016/i.fct.2008.04.040

39 Raben A, Moller AC, Vasilaras TH, et al. A randomized 10 week trial o sucrose vs artificial sweeteners on body weight and blood pressure after 10 weeks[abstr]. Obes Res 2001;9:86s.

40 Reid M, Hammersley R, Duffy M, Ballantyne C. Effects on obese women of the sugar sucrose added to the diet over $28 \mathrm{~d}$ : a quasirandomised, single-blind, controlled trial. Br / Nutr 2014;111:563 70. doi:10.1017/S0007114513002687 
41 Reid M, Hammersley R, Hill AJ, Skidmore P. Long-term dietary compensation for added sugar: effects of supplementary sucrose drinks over a 4-week period. Br J Nutr 2007;97:193-203. doi:10.1017/S0007114507252705

42 Spiers PA, Sabounjian L, Reiner A, Myers DK, Wurtman J, Schomer DL. Aspartame: neuropsychologic and neurophysiologic evaluation of acute and chronic effects. Am J Clin Nutr 1998;68:531-7. doi:10.1093/ajcn/68.3.531

43 van den Eeden SK. A randomized crossover trial of aspartame and sleep. Am J Clin Nutr 1991;53:30.

44 Warrington S, Lee C, Otabe A, et al. Acute and multiple-dose studies to determine the safety, tolerability, and pharmacokinetic profile of advantame in healthy volunteers. Food Chem Toxicol 2011;49(Supp 1):S77-83. doi:10.1016/j.fct.2011.06.043

45 Naismith DJ, Rhodes C. Adjustment in energy-intake following the covert removal of sugar from the diet. I Hum Nutr Diet 1995; 8:16775. doi:10.1111/j.1365-277X.1995.tb00309.x.

46 Porikos KP, Hesser MF, van Itallie TB. Caloric regulation in normalweight men maintained on a palatable diet of conventional foods. Physiol Behav 1982;29:293-300. doi:10.1016/0031 9384(82)90018-X

47 Stanhope K, Bremer A, Medici V, et al. Compared with aspartame, consumption of high fructose corn syrup-and sucrose-sweetened beverages increases triglycerides, cholesterol, non-HDL cholesterol, apolipoprotein-B uric acid in young men and women. J Womens Health (Larchmt) 2013;22:898-9.

48 Stanhope KL, Medici V, Bremer AA, et al. A dose-response study of consuming high-fructose corn syrup-sweetened beverages on lipid/lipoprotein risk factors for cardiovascular disease in young adults. Am J Clin Nutr 2015;101:1144-54. doi:10.3945/ ajcn.114.100461

49 Tordoff MG, Alleva AM. Effect of drinking soda sweetened with aspartame or high-fructose corn syrup on food intake and body weight. Am J Clin Nutr 1990;51:963-9. doi:10.1093/ajcn/51.6.963

50 Colditz GA, Willett WC, Stampfer MJ, London SJ, Segal MR, Speizer FE. Patterns of weight change and their relation to diet in a cohort of healthy women. Am J Clin Nutr 1990;51:1100-5. doi:10.1093/ ajcn/51.6.1100

51 Guo X, Park Y, Freedman ND, et al. Sweetened beverages, coffee, and tea and depression risk among older US adults. PLOS One 2014;9:e94715. doi:10.1371/journal.pone.0094715

52 Lim U, Subar AF, Mouw T, et al. Consumption of aspartamecontaining beverages and incidence of hematopoietic and brain malignancies. Cancer Epidemiol Biomarkers Prev 2006;15:1654-9. doi:10.1158/1055-9965.EPI-06-0203

53 McCullough ML, Teras LR, Shah R, Diver WR, Gaudet MM, Gapstur SM. Artificially and sugar-sweetened carbonated beverage consumption is not associated with risk of lymphoid neoplasms in older men and women. I Nutr 2014:144:2041-9 doi:10.3945/in.114.197475

54 Parker DR, Gonzalez S, Derby CA, Gans KM, Lasater TM, Carleton RA. Dietary factors in relation to weight change among men and women from two southeastern New England communities. Int J Obes Relat Metab Disord 1997:21:103-9. doi:10.1038/sj.ijo.0800373

55 Bosetti C, Gallus S, Talamini R, et al. Artificial sweeteners and the risk of gastric, pancreatic, and endometrial cancers in Italy. Cancer Epidemiol Biomarkers Prev 2009;18:2235-8. doi:10.1158/10559965.EPI-09-0365

56 Cabaniols C, Giorgi R, Chinot O, et al. Links between private habits, psychological stress and brain cancer: a case-control pilot study in France. / Neurooncol 2011;103:307-16. doi:10.1007/s11060-0100388-1

57 Cartwright RA, Adib R, Glashan R, Gray BK. The epidemiology of bladder cancer in West Yorkshire. A preliminary report on non-occupational aetiologies. Carcinogenesis 1981;2:343-7. doi:10.1093/carcin/2.4.343

58 Gallus S, Scotti L, Negri E, et al. Artificial sweeteners and cancer risk in a network of case-control studies. Ann Oncol 2007;18:40-4. doi:10.1093/annonc/mdl346

59 Goodman MT, Morgenstern H, Wynder EL. A case-control study of factors affecting the development of renal cell cancer. Am J Epidemiol 1986;124:926-41. doi:10.1093/oxfordjournals.aje. a114482

60 Howe GR Burch JD, Miller AB, et al. Artificial sweeteners and human bladder cancer. Lancet 1977;310:578-81. doi:10.1016/S01406736(77)91428-3

61 Iscovich J, Castelletto R, Estève J, et al. Tobacco smoking, occupational exposure and bladder cancer in Argentina. Int J Cancer 1987;40:734-40. doi:10.1002/ijc.2910400604

62 Kessler II, Clark JP. Saccharin, cyclamate, and human bladder cancer. No evidence of an association. JAMA 1978;240:349-55. doi:10.1001/jama.1978.03290040027017

63 Møller-Jensen O, Knudsen JB, Sørensen BL, Clemmesen J. Artificial sweeteners and absence of bladder cancer risk in Copenhagen. Int Cancer 1983;32:577-82. doi:10.1002/ijc.2910320510
64 Momas I, Daurès JP, Festy B, Bontoux J, Grémy F. Relative importance of risk factors in bladder carcinogenesis: some new results about Mediterranean habits. Cancer Causes Control 1994:5:326-32. doi:10.1007/BF01804983

65 Najem GR, Louria DB, Seebode JJ, et al. Life time occupation, smoking, caffeine, saccharine, hair dyes and bladder carcinogenesis. Int J Epidemiol 1982;11:212-7. doi:10.1093/ije/11.3.212

66 Nomura AM, Kolonel LN, Hankin JH, Yoshizawa CN. Dietary factors in cancer of the lower urinary tract. Int I Cancer 1991;48:199-205. doi:10.1002/ijc.2910480208. doi:10.1002/ijc.2910480208

67 Simon D, Yen S, Cole P. Coffee drinking and cancer of the lower urinary tract. J Natl Cancer Inst 1975;54:587-91.

68 Yu Y, Hu J, Wang PP, et al. Risk factors for bladder cancer: a casecontrol study in northeast China. Eur J Cancer Prev 1997;6:363-9. doi:10.1097/00008469-199708000-00008

69 Mommsen S, Aagaard J, Sell A. A case-control study of female bladder cancer. Eur J Cancer Clin Oncol 1983;19:725-9. doi:10.1016/02775379(83)90005-6

70 Duran Agüero S, Oñate G, Haro Rivera P. Consumption of non-nutritive sweeteners and nutritional status in 10-16 year old students. Arch Argent Pediatr 2014;112:207-14

71 Fernandes J, Arts J, Dimond E, Hirshberg S, Lofgren IE. Dietary factors are associated with coronary heart disease risk factors in college students. Nutr Res 2013;33:647-52. doi:10.1016/j. nutres.2013.05.013

72 KukJL, Brown RE. Aspartame intake is associated with greater glucose intolerance in individuals with obesity. Appl Physiol Nutr Metab 2016;41:795-8. doi:10.1139/apnm-2015-0675

73 Serra-Majem L, Ribas L, Inglès C, Fuentes M, Lloveras G, Salleras L. Cyclamate consumption in Catalonia, Spain (1992): relationship with the body mass index. Food Addit Contam 1996;13:695-703. doi:10.1080/02652039609374455

74 Wulaningsih W, Van Hemelrijck M, Tsilidis KK, Tzoulaki I, Patel C, Rohrmann S. Investigating nutrition and lifestyle factors as determinants of abdominal obesity: an environment-wide study. Int J Obes (Lond) 2017:41:340-7. doi:10.1038/ijo.2016.203

75 Friel JK. Effects of artificial sweeteners on gut microbiota and glucose metabolism. 2015. https://clinicaltrials.gov/ct2/show/record/ NCT02569762

76 Huber T. Study of the reversibility of glucose intolerance caused by chronic aspartame consumption. 2015. https://clinicaltrials.gov/ct2/ show/record/NCT02520258

77 Kyriazis G. Interactions of human gut microbiota with intestinal sweet taste receptors (ISTAR-micro). 2016. https://clinicaltrials.gov/ct2/ show/record/NCT03032640

78 Mattes RD. Beverage consumption and fine motor control (LCS). 2016. https://clinicaltrials.gov/ct2/show/record/NCT02928653

79 Mattes RD. The effects of aspartame on appetite, body composition and oral glucose tolerance. 2016. https://clinicaltrials.gov/ct2/show/ record/NCT02999321

80 Sriphrapradang C. Sucralose effects on glucose metabolism and gut microbiota. 2016. https://clinicaltrials.gov/ct2/show/record/ NCT02650947

81 Steffen LM. Sucralose, stevia, gut microbiome and glucose metabolism. 2016. https://clinicaltrials.gov/ct2/show/record/ NCT02800707

82 Nilsson A. On the impact of common sweetening agents on glucose regulation, cognitive functioning and gut microbiota. 2015. https:// clinicaltrials.gov/ct2/show/record/NCT02580110

83 Wolraich ML, Lindgren SD, Stumbo PJ, Stegink LD, Appelbaum MI, Kiritsy MC. Effects of diets high in sucrose or aspartame on the behavior and cognitive performance of children. N Engl J Med 1994:330:301-7. doi:10.1056/NEJM199402033300501

84 de Ruyter JC, Katan MB, Kuijper LD, Liem DG, Olthof MR. The effect of sugar-free versus sugar-sweetened beverages on satiety, liking and wanting: an 18 month randomized double-blind tria in children. PLoS One 2013;8:e78039. doi:10.1371/journal. pone.0078039

85 Frey GH. Use of aspartame by apparently healthy children and adolescents. / Toxicol Environ Health 1976:2:401-15. doi:10.1080/15287397609529442

86 Knopp RH, Brandt K, Arky RA. Effects of aspartame in young persons during weight reduction. I Toxicol Environ Health 1976;2:417-28. doi:10.1080/15287397609529443

87 Taljaard C, Covic NM, van Graan AE, et al. Effects of a multimicronutrient-fortified beverage, with and without sugar, on growth and cognition in South African schoolchildren: a randomised, double-blind, controlled intervention. Br J Nutr 2013;110:2271-84. doi:10.1017/S000711451300189X

88 Zanela NL, Bijella MF, Rosa OP. The influence of mouthrinses with antimicrobial solutions on the inhibition of dental plaque and on the levels of mutans streptococci in children. Pesqui Odontol Bras 2002;16:101-6. doi:10.1590/S1517 74912002000200002 
89 Gurney JG, Pogoda JM, Holly EA, Hecht SS, Preston-Martin S. Aspartame consumption in relation to childhood brain tumor risk: results from a case-control study. I Natl Cancer Inst 1997;89:1072-4. doi:10.1093/jnci/89.14.1072

90 Small DM. The effect of artificial sweeteners (AFS) on sweetness sensitivity, preference and brain response in adolescents. 2015. https://clinicaltrials.gov/ct2/show/NCT02499705

91 de Ruyter JC, Olthof MR, Kuijper LD, Katan MB. Effect of sugarsweetened beverages on body weight in children: design and baseline characteristics of the Double-blind, Randomized INtervention study in Kids. Contemp Clin Trials 2012;33:247-57. doi:10.1016/i.cct.2011.10.007

92 de Ruyter JC, Olthof MR, Seidell JC, Katan MB. A trial of sugar-free or sugar-sweetened beverages and body weight in children. N Engl J Med 2012;367:1397-406. doi:10.1056/NEJMoa1203034

93 Lohner S, Toews I, Meerpohl JJ. Health outcomes of non-nutritive sweeteners: analysis of the research landscape. Nutr J 2017;16:55. doi:10.1186/s12937-017-0278-x

94 Bes-Rastrollo M, Schulze MB, Ruiz-Canela M, Martinez-Gonzalez MA. Financial conflicts of interest and reporting bias regarding the association between sugar-sweetened beverages and weight gain: a systematic review of systematic reviews. PLoS Med 2013;10:e1001578, e1001578 doi:10.1371/journal.pmed.1001578

95 Onakpoya IJ, Heneghan CJ. Effect of the natural sweetener, steviol glycoside, on cardiovascular risk factors: a systematic review and meta-analysis of randomised clinical trials. Eur J Prev Cardiol 2015;22:1575-87. doi:10.1177/2047487314560663

96 Ulbricht C, Isaac R, Milkin T, et al. An evidence-based systematic review of stevia by the Natural Standard Research Collaboration. Cardiovasc Hematol Agents Med Chem 2010;8:113-27. doi:10.2174/187152510791170960

97 Azad MB, Abou-Setta AM, Chauhan BF, et al. Nonnutritive sweeteners and cardiometabolic health: a systematic review and meta-analysis of randomized controlled trials and prospective cohort studies. CMAJ 2017;189:E929-39. doi:10.1503/cmaj.161390

98 Chia CW, Shardell M, Tanaka T, et al. Chronic low-calorie sweetener use and risk of abdominal obesity among older adults: A cohort study. PLoS One 2016;11:e0167241. doi:10.1371/journal.pone.0167241

99 Drewnowski A, Rehm CD. The use of low-calorie sweeteners is associated with self-reported prior intent to lose weight in a representative sample of US adults. Nutr Diabetes 2016;6:e202-02 doi:10.1038/nutd.2016.9

100 Fagherazzi G, Gusto G, Affret A, et al. Chronic consumption of artificial sweetener in packets or tablets and type 2 diabetes risk: evidence from the E3N-European Prospective Investigation into Cancer and Nutrition Study. Ann Nutr Metab 2017;70:51-8. doi:10.1159/000458769

101 Stepien M, Duarte-Salles T, Fedirko V, et al. Consumption of soft drinks and juices and risk of liver and biliary tract cancers in a European cohort. Eur J Nutr 2016;55:7-20. doi:10.1007/s00394-014-0818-5

102 de Koning L, Malik VS, Kellogg MD, Rimm EB, Willett WC Hu FB. Sweetened beverage consumption, incident coronary heart disease, and biomarkers of risk in men. Circulation 2012;125:1735-41, S1. doi:10.1161/ CIRCULATIONAHA.111.067017

103 Wan X, Wang W, Liu J, Tong T. Estimating the sample mean and standard deviation from the sample size, median, range and/ or interquartile range. BMC Med Res Methodol 2014;14:135 doi:10.1186/1471-2288-14-135

104 Lohner S, Toews I, Kuellenberg de Gaudry D, et al. Non-nutritive sweeteners for diabetes mellitus. Cochrane Database Syst Rev 2017;11:CD012885

105 Sylvetsky AC, Blau JE, Rother KI. Understanding the metabolic and health effects of low-calorie sweeteners: methodological considerations and implications for future research. Rev Endocr Metab Disord 2016;17:187-94. doi:10.1007/s11154016-9344-5

106 Sievenpiper JL, Khan TA, Ha V, Viguiliouk E, Auyeung R. The importance of study design in the assessment of nonnutritive sweeteners and cardiometabolic health. CMAI 2017;189:E1424-5. doi:10.1503/cmaj.733381

Supplementary file 1: Supplementary materials Supplementary file 2: Results of the assessment of risk of bias in included observational studies Supplementary file 3: Details of included studies (RCT=randomised, controlled trial; nonRCT=non-randomised controlled trial; $A S=$ artificial sweetener, $\mathrm{CVD}=$ cardiovascular disease); ${ }^{\star}$ For profit funding includes sponsoring of study material, i.e. intervention substances, as well as financial sponsoring for conducting the study 